\title{
(6) OPEN ACCESS \\ Nomogram incorporating clinicopathological parameters to predict the survival of patients with mantle cell lymphoma
}

\author{
Yuandong Zhu, ${ }^{1}$ Wenxian $\mathrm{Xu}_{1}{ }^{2}$ Xiao Zheng, ${ }^{3}$ Zhuojun Zheng ${ }^{1}$
}

'Department of Hematology, The Third Affiliated Hospital of Soochow University,

Changzhou, China

${ }^{2}$ Department of Pathology, The First Affiliated Hospital of Soochow University, Suzhou, China ${ }^{3}$ Department of Tumor Biological Treatment, The Third Affiliated Hospital of Soochow University, Changzhou, China

\section{Correspondence to} Xiao Zheng, Department of Tumor Biological Treatment The Third Affiliated Hospital of Soochow University, Juqian Road No.185, Changzhou 213003, China; zhengxia0923@163.com and Dr Zhuojun Zheng, Department of Hematology, The Third Affiliated Hospital of Soochow University, Changzhou 213003, China; zenki_zheng@163.com

Accepted 29 August 2018 Published Online First 25 September 2018

\section{Check for updates}

(C) American Federation for Medical Research 2019. Re-use permitted under CC BY-NC. No commercial re-use. Published by BMJ.

To cite: Zhu Y, XuW, Zheng X, etal.JInvestigMed 2019;67:331-337.

\section{ABSTRACT}

This study intended to present a practicable prognostic nomogram for patients with mantle cell lymphoma (MCL). The clinical data of 281 patients were reviewed. A nomogram that could predict overall survival (OS) was constructed based on the Cox proportional hazard model. To compare the capacity of the nomogram with the International Prognostic Index (IPI) and MCL International Prognostic Index (MIPI) scoring systems, we used the concordance index (C-index) to validate the veracity and the calibration curve. Age, Eastern Cooperation Oncology Group, lactate dehydrogenase, white cell count and Ki-67 were independent prognostic factors in the multivariate analysis and were subsequently included in the nomogram construction. The C-index was 0.81 and 0.79 in the primary and validation cohorts, respectively, which were superior to the predictive capacity of the IPI and MIPI systems in both cohorts. The nomogram makes it possible for physicians to predict patient OS individually and correctly, but certain limitations are noted.

\section{INTRODUCTION}

Mantle cell lymphoma (MCL) is a highly aggressive subtype of medium or small B cell type non-Hodgkin's lymphoma that originates from the inner follicle mantle and is characterized by lymph node, gastrointestinal tract, bone marrow (BM)and peripheral blood infiltration. The estimated annual incidence of MCL is only 0.8 cases per 100000 person-years in the USA since 2001 according to Surveillance, Epidemiology, and End Results rates. MCL exhibits CCND1 gene overexpression, and a new disease entity is typically characterized by $\mathrm{t}(11 ; 14)(\mathrm{q} 13 ; \mathrm{q} 32) .{ }^{1}$ The disease often occurs in middle-aged and senile men, most of whom are diagnosed at stages III-IV with diffuse involvement of extranodal sites. ${ }^{2}$

It is difficult to determine the optimal prognostic model for MCL given its rarity and heterogeneity. Various prognostic factors are associated with survival in MCL. Elevated lactate dehydrogenase (LDH), older age, advanced stage, a senior score in the International Prognostic Index (IPI) scoring system, presence of B symptoms, poor performance

\section{Significance of this study}

What is already known about this subject?

- Mantle cell lymphoma (MCL) is a highly aggressive subtype of medium or small B-cell type non-Hodgkin's lymphoma. Currently, it remains difficult to determine the optimal prognostic model for MCL. The nomogram is an advanced prediction model.

What are the new findings?

- Age, EOCG, LDH, WBC and Ki-67 index were included in nomogram. The nomogram could predict the overall survival (OS) of patients with MCL more precisely than the current scoring system. The nomogram passed external validation.

How might these results change the focus of research or clinical practice?

- The nomogram makes it possible for physicians to predict patient OS individually. The nomogram provides a simple visual format for all users.

status (Eastern Cooperation Oncology Group $(\mathrm{ECOG}))$, and high mitotic index (Ki-67) are associated with poor prognosis. ${ }^{34}$ Recently, the MCL International Prognostic Index (MIPI) scoring system was established by Hoster. ${ }^{5}$ This system divides patients with MCL into groups by incorporating age, performance status, LDH level, and white cell count (WCC) to predict the overall survival (OS). For each prognostic factor, 0-3 points are given to each patient, and points are added to yield a maximum of 11 points. Patients with a total of $0-3$ points are classified as low risk, patients with 4-5 points are classified as intermediate risk, and patients with 6-11 points are classified as high risk in the simplified MIPI score system. ${ }^{5}$ However, this classification remains uncertain when taking racial differences into consideration, and thus, further validation is required.

The nomogram is an advanced prediction model that provides a simple visual format. The nomogram could estimate survival by integrating diverse variables. The nomogram has been proved to be acceptable for several types 
Table 1 Baseline characteristics of patients

\begin{tabular}{|c|c|c|c|c|}
\hline \multirow[b]{2}{*}{ Characteristic } & \multicolumn{2}{|c|}{ Primary cohort $(n=198)$} & \multicolumn{2}{|c|}{ Validation cohort $(n=83)$} \\
\hline & Patients (n) & $\%$ & Patients (n) & $\%$ \\
\hline \multicolumn{5}{|l|}{ Sex } \\
\hline Male & 162 & 81.8 & 62 & 74.7 \\
\hline Female & 36 & 18.2 & 21 & 25.3 \\
\hline \multicolumn{5}{|l|}{ Age } \\
\hline$<50$ & 9 & 4.5 & 4 & 4.8 \\
\hline $50-59$ & 36 & 18.2 & 17 & 20.5 \\
\hline $60-69$ & 54 & 27.3 & 21 & 25.3 \\
\hline$\geq 70$ & 99 & 50.0 & 41 & 49.4 \\
\hline \multicolumn{5}{|l|}{ ECOG } \\
\hline $0-1$ & 117 & 59.0 & 42 & 50.6 \\
\hline $2-4$ & 81 & 41.0 & 41 & 49.4 \\
\hline \multicolumn{5}{|l|}{ LDH/ULN } \\
\hline$<0.67$ & 76 & 38.4 & 29 & 34.9 \\
\hline $0.67-0.99$ & 59 & 29.8 & 28 & 33.7 \\
\hline $1.0-1.49$ & 23 & 11.6 & 16 & 19.3 \\
\hline$\geq 1.5$ & 40 & 20.2 & 10 & 12.0 \\
\hline \multicolumn{5}{|l|}{ WCC $\left(\times 10^{9} / L\right)$} \\
\hline$<5.00$ & 35 & 17.7 & 16 & 19.3 \\
\hline $5.00-9.99$ & 54 & 27.3 & 25 & 30.1 \\
\hline $10.00-14.99$ & 64 & 32.3 & 23 & 27.7 \\
\hline$\geq 15.00$ & 45 & 22.7 & 19 & 22.9 \\
\hline \multicolumn{5}{|l|}{ Ki-67 index } \\
\hline$<30 \%$ & 72 & 36.4 & 33 & 39.8 \\
\hline$\geq 30 \%$ & 126 & 63.6 & 50 & 60.2 \\
\hline \multicolumn{5}{|l|}{$\beta 2$-microglobulin } \\
\hline Normal & 108 & 54.5 & 41 & 49.4 \\
\hline Elevated & 90 & 45.5 & 42 & 50.6 \\
\hline \multicolumn{5}{|l|}{ B symptoms } \\
\hline Yes & 97 & 49.0 & 40 & 48.2 \\
\hline No & 101 & 51.0 & 43 & 51.8 \\
\hline \multicolumn{5}{|l|}{ Extranodal sites } \\
\hline$<2$ & 79 & 39.9 & 29 & 34.9 \\
\hline$\geq 2$ & 119 & 60.1 & 54 & 65.1 \\
\hline \multicolumn{5}{|l|}{$\begin{array}{l}\text { Bone marrow } \\
\text { involvement }\end{array}$} \\
\hline Yes & 72 & 36.4 & 24 & 28.9 \\
\hline No & 126 & 63.6 & 49 & 71.1 \\
\hline \multicolumn{5}{|l|}{ Ann Arbor stage } \\
\hline III & 45 & 22.7 & 17 & 20.5 \\
\hline IV & 153 & 77.3 & 66 & 79.5 \\
\hline \multicolumn{5}{|l|}{$\begin{array}{l}\text { Treatment } \\
\text { regimen }\end{array}$} \\
\hline $\mathrm{R}-\mathrm{CHOP}$ & 107 & 54.0 & 46 & 55.4 \\
\hline Other & 91 & 46.0 & 37 & 44.6 \\
\hline \multicolumn{5}{|l|}{ IPI score } \\
\hline$<3$ & 72 & 30.3 & 22 & 26.5 \\
\hline$\geq 3$ & 126 & 69.7 & 61 & 73.5 \\
\hline \multicolumn{5}{|l|}{ MIPI score } \\
\hline $0-3$ & 45 & 22.7 & 14 & 16.9 \\
\hline $4-5$ & 72 & 36.4 & 30 & 36.1 \\
\hline $6-11$ & 81 & 40.9 & 39 & 47.0 \\
\hline
\end{tabular}

ECOG, Eastern Cooperation Oncology Group; IPI, International Prognostic Index; LDH, lactate dehydrogenase; MIPI, Mantle cell lymphoma International Prognostic Index; R-CHOP, rituximab, cyclophosphamide, doxorubicin, and prednisone; ULN, upper limits of normal; WCC, white cell count. of cancers and serves as an important alternative for physicians under certain conditions. ${ }^{6-11}$

This study sought to perform a prognostic nomogram for MCL based on the clinicopathological parameters compared with the IPI and MIPI.

\section{MATERIALS AND METHODS Patient data}

A retrospective review of the medical records of 198 patients who were all newly diagnosed as MCL by histological biopsy between January 2002 and March 2014 at the First Affiliated Hospital of Soochow University was conducted. An additional 83 patients diagnosed from January 2002 to December 2015 at the Third Affiliated Hospital of Soochow University were enrolled in the external validation cohort. The WHO classification of lymphoid neoplasms version 2008 was applied as histological criteria. ${ }^{12}$ All patients were followed up to November 2016. All patients were evaluated and staged according to the Ann Arbor Staging classification, which includes CT and BM aspirate data. Age, sex, ECOG grade, Ann Arbor stage, presence of extranodal disease, WCC, serum LDH, serum $\beta 2$-microglobulin, Ki-67 index, IPI score, simplified MIPI score, result of BM aspirate and biopsy were collected. All patients received chemotherapy mainly based on the recommendation by National Comprehensive Cancer Network (NCCN) Clinical Practice Guidelines in Oncology: Non-Hodgkin's Lymphomas. Approximately, half of patients received rituximab, cyclophosphamide, doxorubicin, vincristine and prednisone $(\mathrm{R}+\mathrm{CHOP})$ or cyclophosphamide, doxorubicin, and prednisone (CHOP)-like regimens as primary therapeutic approaches. The other patients received chemotherapy regimens including but not limited to rituximab, etoposide, methylprednisone, cytarabine and cisplatin (RESHAP), doxorubicin, vincristine, dexamethasone and cytarabine (R+HyperCVAD), CHOP, fludarabine, bortezomib and thalidomide.

The study protocol was designed in accordance with the guidelines outlined in the Declaration of Helsinki. Written informed consent was obtained from all patients.

\section{Statistical analysis}

SPSS V.22.0 and R project V.3.2.2 (http://www.r-project. org/) with Hmisc, rms and survival receiver operating characteristic (ROC) packages were used for statistical analysis. OS represents the time from the date of diagnosis to the date of death. The proportion was used to describe the descriptive statistics. Univariate and multivariate survival analyses were calculated using log-rank test (Kaplan-Meier) and Cox's regression model. The nomogram was established according to multivariate analysis. Backward step-down selection processed the final model selection. Discrimination and calibration were performed to evaluate the nomogram capacity. The ROC curve and C-index were calculated to validate the discrimination power for OS among different models. Over 1000 bootstrap samples were created to estimate CIs and replicated the estimation process. The larger the $\mathrm{C}$-index value, the more accurate the prediction. To externally validate the nomogram, we calculated the 
Table 2 Univariate and multivariate cox regression analyses for overall survival in patients with mantle cell lymphoma

\begin{tabular}{|c|c|c|c|c|}
\hline \multirow[b]{2}{*}{ Variables } & \multicolumn{2}{|l|}{ Univariate analysis } & \multicolumn{2}{|l|}{ Multivariate analysis } \\
\hline & HR $(95 \% \mathrm{Cl})$ & $P$ values & HR $(95 \% \mathrm{Cl})$ & $P$ values \\
\hline \multicolumn{5}{|l|}{ Sex } \\
\hline Female VS Male & 0.60 (0.39 to 0.93$)$ & 0.023 & $0.58(0.25$ to 1.35$)$ & 0.208 \\
\hline Age & & $<0.001$ & & $<0.001$ \\
\hline$<50$ & Reference & & Reference & \\
\hline $50-59$ & 5.95 (1.34 to 26.46$)$ & 0.019 & 11.15 (1.43 to 87.25$)$ & 0.022 \\
\hline $60-69$ & $3.35(0.80$ to 14.02$)$ & 0.098 & $10.49(1.15$ to 95.91$)$ & 0.037 \\
\hline$\geq 70$ & 14.88 (3.50 to 63.34$)$ & $<0.001$ & 12.65 (1.74 to 91.95$)$ & 0.012 \\
\hline \multicolumn{5}{|l|}{ EOCG } \\
\hline $0-1$ versus $2-4$ & 4.30 (2.86 to 6.47$)$ & $<0.001$ & 14.21 (4.67 to 43.25 ) & $<0.001$ \\
\hline LDH/ULN & & $<0.001$ & & $<0.001$ \\
\hline$<0.67$ & Reference & & Reference & \\
\hline $0.67-0.99$ & 2.86 (1.83 to 4.47$)$ & $<0.001$ & 18.81 (6.84 to 51.71$)$ & $<0.001$ \\
\hline $1.0-1.49$ & 1.67 (0.95 to 2.93$)$ & 0.074 & 9.47 (3.21 to 27.99$)$ & $<0.001$ \\
\hline$\geq 1.5$ & 5.08 (3.12 to 8.25$)$ & $<0.001$ & 46.67 (15.95 to 136.57$)$ & $<0.001$ \\
\hline WCC $\left(\times 10^{9} / L\right)$ & & 0.001 & & 0.040 \\
\hline$<6.70$ & Reference & & Reference & \\
\hline $6.70-9.99$ & 2.71 (1.45 to 5.08$)$ & 0.002 & 3.04 (0.95 to 9.74$)$ & 0.062 \\
\hline $10.00-14.99$ & 3.30 (1.81 to 6.02$)$ & 0.110 & 1.74 (0.81 to 3.73$)$ & 0.154 \\
\hline$\geq 15.00$ & 3.31 (1.76 to 6.19$)$ & $<0.001$ & 3.02 (1.37 to 6.62$)$ & 0.006 \\
\hline \multicolumn{5}{|l|}{ Ki-67 index } \\
\hline$\geq 30 \%$ versus $<30 \%$ & 6.02 (3.19 to 11.34$)$ & $<0.001$ & 3.20 (1.16 to 8.82 ) & 0.024 \\
\hline \multicolumn{5}{|l|}{$\beta 2$-microglobulin } \\
\hline Elevated versus normal & 1.53 (1.07 to 2.18$)$ & 0.019 & 1.16 (0.66 to 8.82$)$ & 0.604 \\
\hline \multicolumn{5}{|l|}{ B symptoms } \\
\hline Yes versus no & 1.27 (0.93 to 1.71$)$ & 0.129 & & \\
\hline \multicolumn{5}{|l|}{ Extranodal sites } \\
\hline$\geq 2$ versus $<2$ & 2.44 (1.66 to 3.60$)$ & $<0.001$ & 1.93 (1.37 to 2.72 ) & 0.236 \\
\hline \multicolumn{5}{|l|}{ Bone marrow involvement } \\
\hline Yes versus no & 5.52 (3.63 to 8.41$)$ & $<0.001$ & 1.82 (0.85 to 3.88$)$ & 0.125 \\
\hline \multicolumn{5}{|l|}{ Ann Arbor stage } \\
\hline IV versus III & 2.18 (1.41 to 3.35$)$ & $<0.001$ & 1.31 (0.71 to 2.44$)$ & 0.386 \\
\hline \multicolumn{5}{|l|}{ Treatment regimen } \\
\hline $\mathrm{R}-\mathrm{CHOP}$ versus other & 0.70 (0.49 to 1.02$)$ & 0.060 & & \\
\hline
\end{tabular}

ECOG, Eastern Cooperation Oncology Group; IPI, International Prognostic Index; LDH, lactate dehydrogenase; MIPI, Mantle cell lymphoma International Prognostic; R-CHOP, rituximab, cyclophosphamide, doxorubicin, and prednisone; ULN, upper limits of normal; WCC, white cell count.

total points for each patient in the validation cohort as well as the C-index and calibration curve. Iasonos' guide was strictly obeyed during nomogram construction and validation. ${ }^{13}$ Differences were considered statistically significant when $\mathrm{p}<0.05$.

\section{RESULTS}

\section{Clinical features and characteristics}

In total, 198 patients were included in the primary cohort in this study (table 1). The median age was 69 years (range 23-86 years), and 162 patients were male. A total of 153 patients $(77.3 \%)$ were classified as Ann Arbor stage IV at diagnosis. The overall incidence of extranodal involvement (greater than two sites) was $60.1 \%$. An elevated Ki-67 index $(\geq 30 \%)$ was noted in 126 patients $(63.6 \%)$. According to the simplified MIPI, 81 patients (40.1\%) were at high risk. Patients were followed up until death or March 2015. The median OS was 19.5 months (range, 1-60 months). OS values at 1 year, 2 years and 3 years were $45.45 \%$, 31.82\% and $18.18 \%$, respectively. The other clinicopathological characteristics of patients are listed in table 1 .

\section{Nomogram development and internal validation}

We used the primary cohort to build the nomogram. In univariate analysis, sex, age, ECOG, LDH, WCC, Ki-67 index, $\beta 2$-microglobulin, extranodal sites, BM involvement and Ann Arbor stage were identified as independent prognostic factors, whereas B symptoms and treatment regimen exhibited no significant differences (table 2). Multivariate analyses confirmed that age, ECOG, LDH, WCC and Ki-67 index were independent risk factors (table 2). Age, ECOG score, LDH, Ki-67 and WCC exhibited statistically significant effects on OS based on log-rank test (all p<0.001) (figure 1). To determine the best-fit model among independent risk factors, we performed backward stepwise selection with the Akaike information criterion in Cox modeling. Finally, five variables were included in the nomogram. Age, ECOG, LDH, WCC and Ki-67 index were used to predict 
A
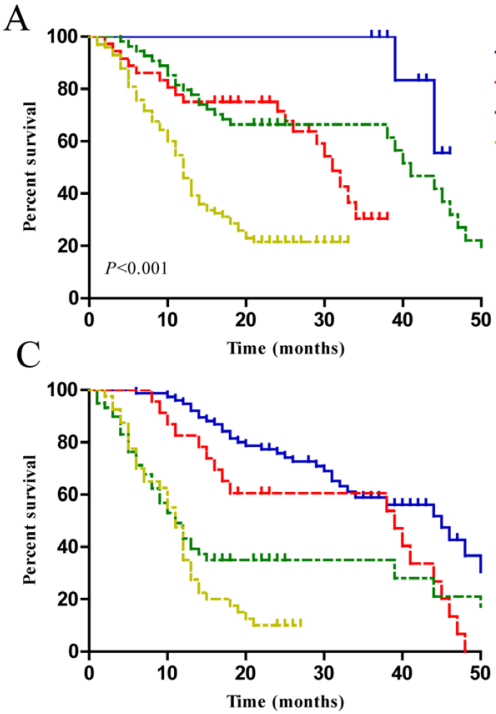

+ $\mathrm{LDH} / \mathrm{ULR}<0.67$

-L. LDH/ULR 0.67-0.99

.... LDH/ULR 1.0-1.49

L. LDH/ULR $\geq 1.50$
B

$\mathrm{D}$
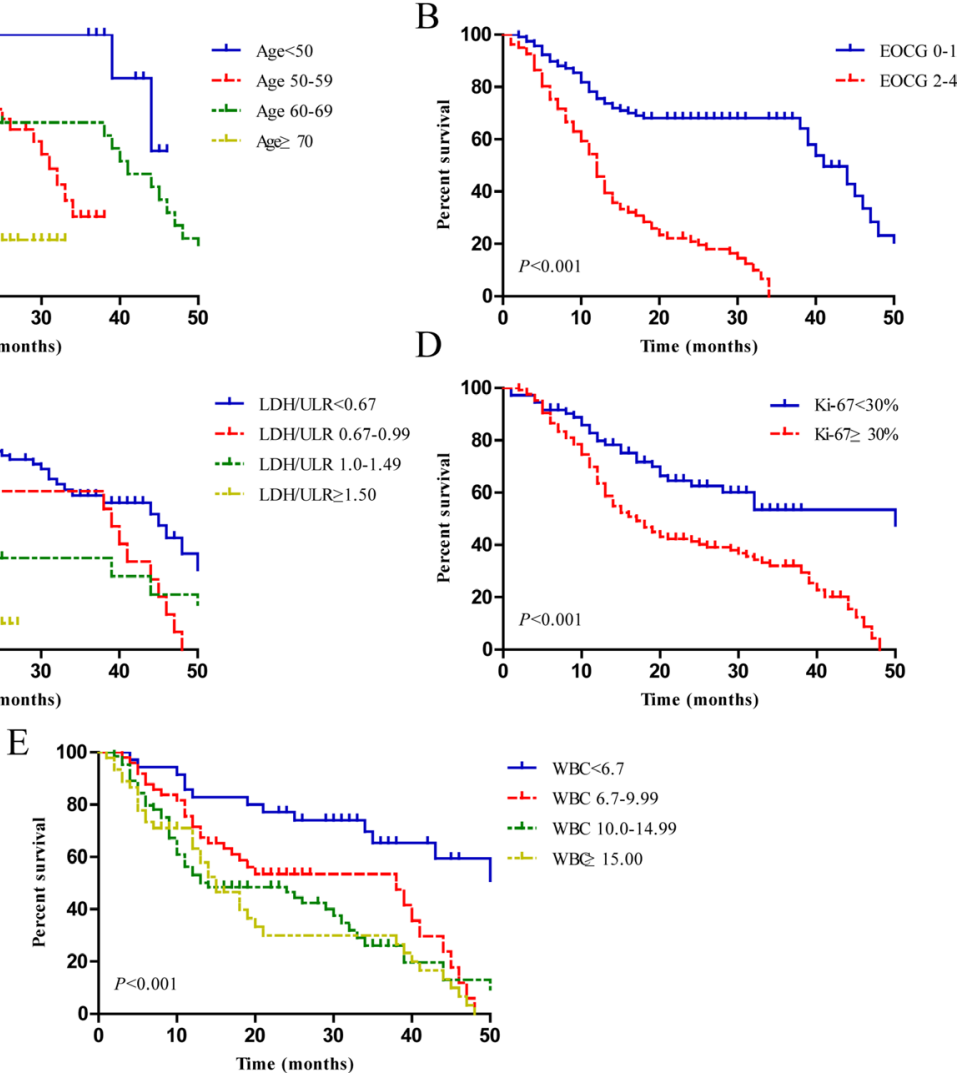

Figure 1 Overall survival according to (A) age, (B) Eastern Cooperation Oncology Group (ECOG) scores, (C) serum lactate dehydrogenase (LDH), (D) Ki-67, and (E) white cell count (WCC).

Points
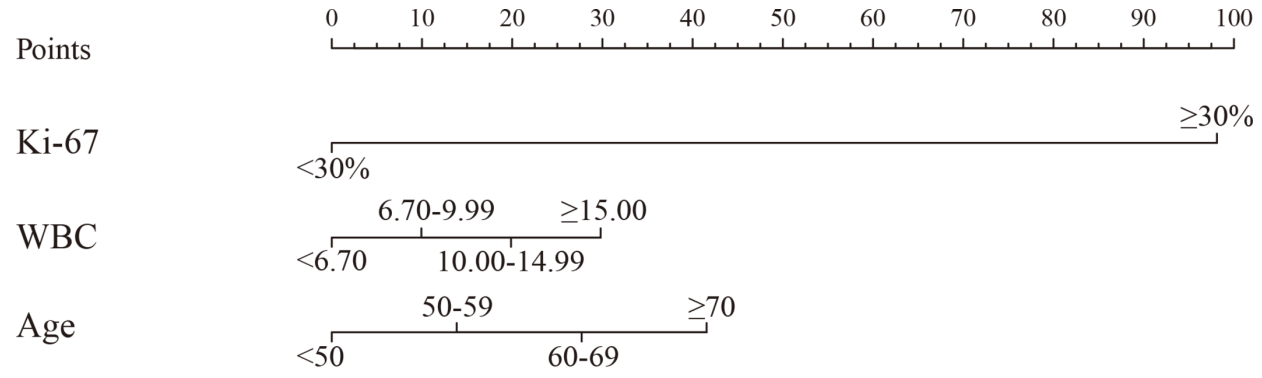

EOCG

LDH/ULR

$$
0-1
$$

$<0.67 \quad 0.67-0.99 \quad \geq 1.5$

Total Points

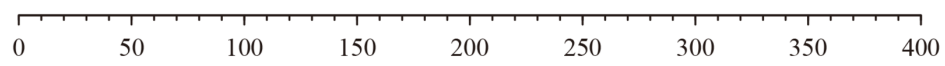

1-year survival

\begin{tabular}{lllllllllllll}
\hline & & & & & & & & & & & & \\
0.9 & 0.85 & 0.80 .750 .7 & 0.6 & 0.5 & 0.4 & 0.3 & 0.2 & 0.1
\end{tabular}

3-year survival

\begin{tabular}{lllllllllllll}
\hline & 0.9 & 0.85 & 0.80 .750 .7 & 0.6 & 0.5 & 0.4 & 0.3 & 0.2 & 0.1
\end{tabular}

Figure 2 Nomogram evaluation in the patients with mantle cell lymphoma. To use the nomogram, the value attributed to an individual patient is located on each variable axis, and a line is drawn upwards to determine the number of points received for each variable value. To determine the 1-year, 2-year and 3-year overall survival probability, a line is drawn downwards from the total points' axis, where the total points represent a sum of points of each variable, to the survival axis. ECOG, Eastern Cooperation Oncology Group; LDH, lactate dehydrogenase; ULN, upper limits of normal; WCC, white cell count. 


\section{A}

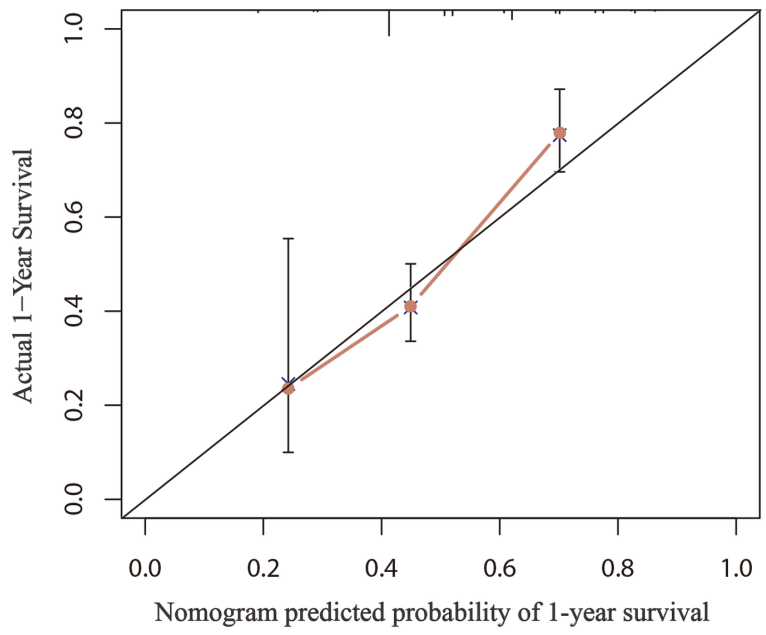

B

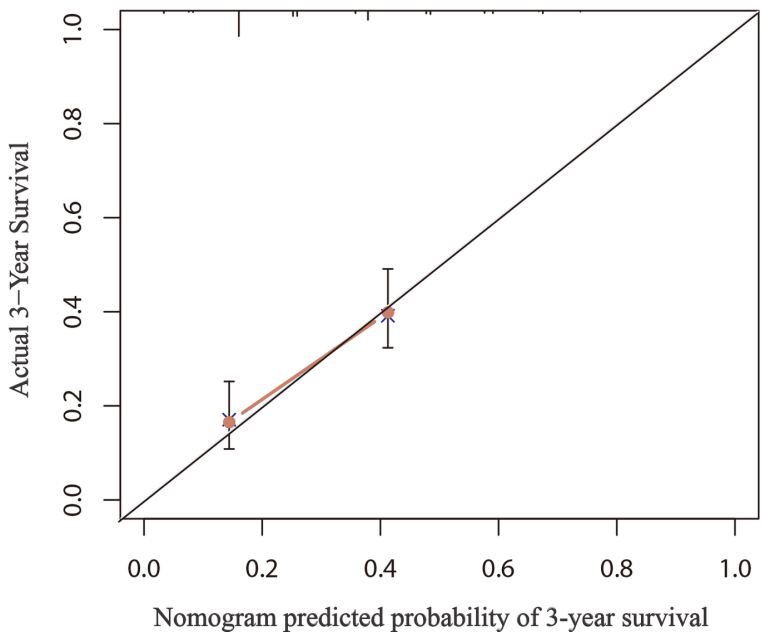

Figure 3 The calibration curve for predicting patient survival at (A) 1 year and (B) 3 years in the primary cohort.

1-year and 3-year OS in MCL (figure 2). The C-index was 0.81 (95\% CI 0.78 to 0.84 ). The calibration plot of the nomogram prediction and actual observation for the probability of 1-year to 3-year survival exhibited good consistency (figure $3 \mathrm{~A}, \mathrm{~B}$ ). The C-index of the nomogram was increased compared with the IPI scoring system (0.69) and MIPI scoring system $(0.76)(\mathrm{p}<0.001)$. The time-dependent ROC curve exhibited an improved capacity for predicting 1-year, 2-year and 3-year OS (figure 4A-C). These results suggest that our nomogram exhibits better performance for predicting OS compared with IPI and MIPI.

\section{Validation of the nomogram for OS}

The nomogram was applied to each patient in the validation cohort. Favorable consistency in 1-year and 3-year survival between actual observation and nomogram prediction was observed based on calibration curves (figure $5 \mathrm{~A}$, B). The C-index was 0.79 (95\%CI 0.75 to 0.84 ) in the validation cohort, which was also better than the IPI scoring system $(0.68)(p<0.001)$ and MIPI scoring system $(0.76)$ $(\mathrm{p}<0.001)$. The similar results were also noted for the ROC (figure 6A-C).

\section{DISCUSSION}

MCL, which is characterized by frequent relapse and a poor prognosis, is a rare type of non-Hodgkin's lymphoma. Recent studies revealed two clinical subtype classifications based on disparities in pathogenesis. ${ }^{14} 15$ The classical MCL originated from mature B cells without developing from the follicular germinal centre and somatic immunoglobulin heavy chain variable mutation. Recent studies demonstrated that MCL commonly occurs in elderly men (median age 63 years), most of whom are diagnosed at an advanced stage. BM involvement was common. ${ }^{16-18}$ Similar clinical characteristics were observed in this study. No standard chemotherapeutic regimen has been identified; however, various regimens exhibit significant improvements in patients with MCL.

Diverse therapeutic approaches and disease heterogeneity have made it difficult to establish a favorable
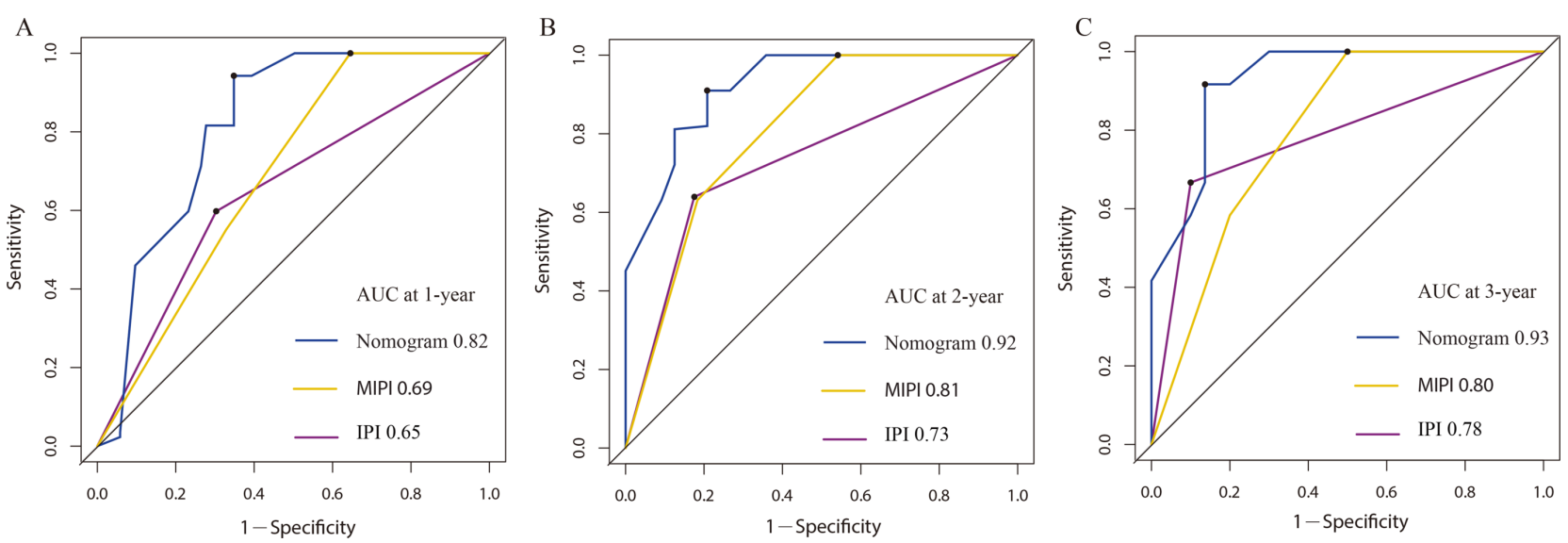

Figure 4 Time-dependent receiver operating characteristic curves from the nomogram, IPI and MIPI for (A) 1-year, (B) 2-year and (C) and 3-year overall survival in the primary cohort. AUC, area under the curve; IPI, International Prognostic Index; MIPI, Mantle cell lymphoma International Prognostic Index. 

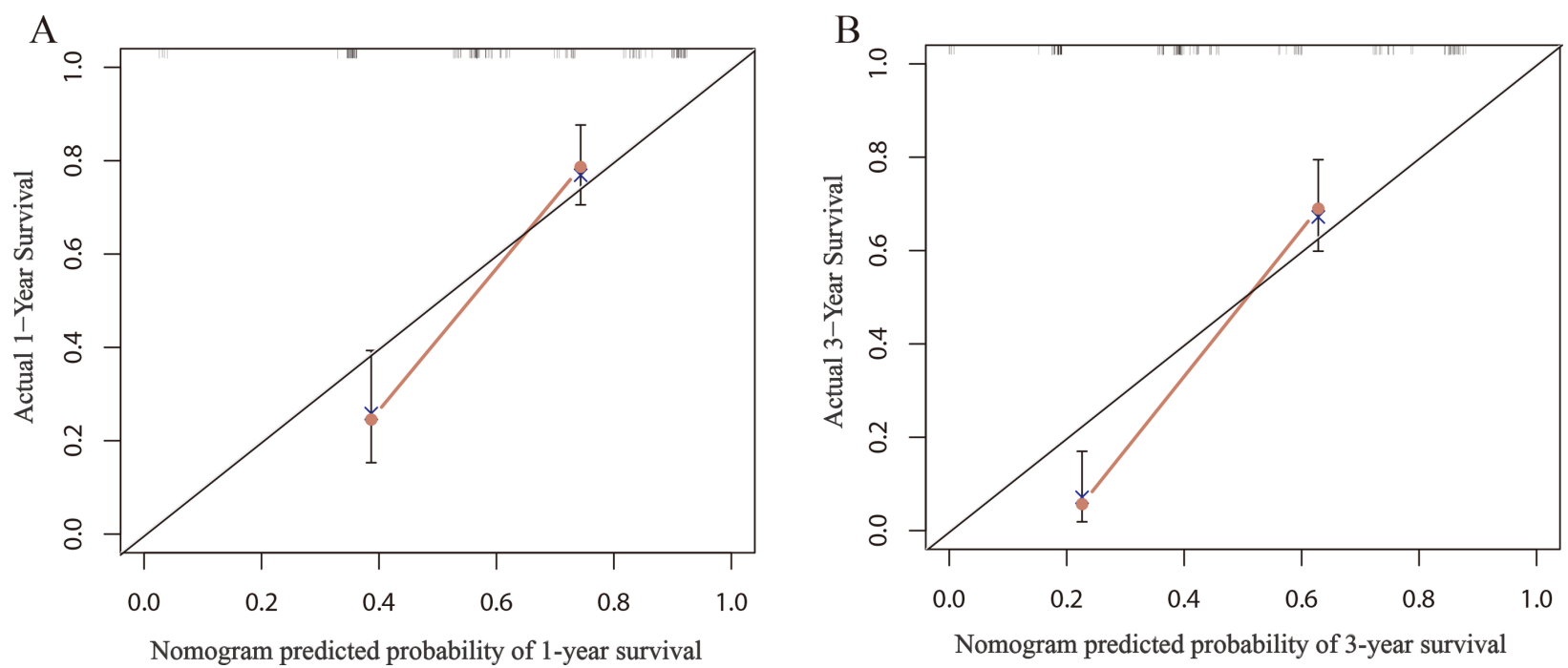

Figure 5 The calibration curve for predicting patient survival at (A) 1 year and (B) 3 year in the validation cohort.

predictive mode. Some groups have made efforts and achievements. ${ }^{19-21}$ The variables included in the nomogram are also recommended by NCCN Clinical Practice Guidelines in Oncology: Non-Hodgkin"s Lymphomas. Thus, the nomogram is practical for most medical institutions. Nomogram development and validation was accomplished at two independent institutions. In our nomogram, some results were unexpected. Some risk factors highlighted in NCCN Clinical Practice Guidelines in Oncology: Non-Hodgkin's Lymphomas, such as B symptom, BM and extranodal involvement and advanced Ann Arbor stage, were not associated with survival in the multivariate Cox regression analysis. MCL exhibits different clinical features compared with other NHLs, which may partially explain the differences. In addition, all patients included were diagnosed at III or IV stage, which are regarded as advanced stage. Not surprisingly, stratification by Ann Arbor stage cannot accurately predict prognosis.

The nomogram offers more benefits compared with the current existing prognostic system in several clinical settings as it integrates different risk factors to provide an individualized assessment. ${ }^{22-27}$ It also exhibited good predictive ability for OS compared with some of the current prognostic systems in this study. The IPI scoring system exhibited a good ability to predict OS (C-index: 0.69 in the primary cohort, 0.68 in the validation cohort). The MIPI scoring system was even better than IPI (C-index: 0.76 in both cohorts). The nomogram exhibited the best accuracy in prognostic prediction among the three models. The results were solidly consolidated according to the area under curve. As expected, the inclusion of more independent factors made the nomogram more powerful compared with the existing prognostic systems. With the exception of variables also included in the MIPI system, we further consolidated the important prognostic role of the $\mathrm{Ki}-67$ index, which has also been reported in MCL by Hoster. ${ }^{28}$ To some extent, the nomogram could be regarded as an updated version of the MIPI scoring system, including the addition of a visualization and interactive interface. To the best of our knowledge, no other nomograms concerning MCL have been published to date given that it is difficult to collect sufficient cases from a single institution.
A

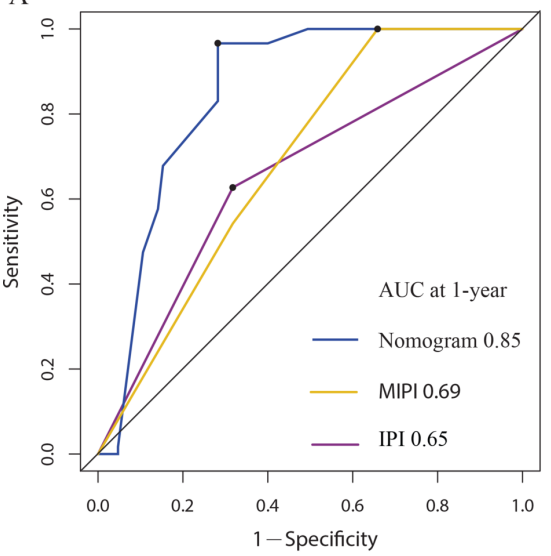

B

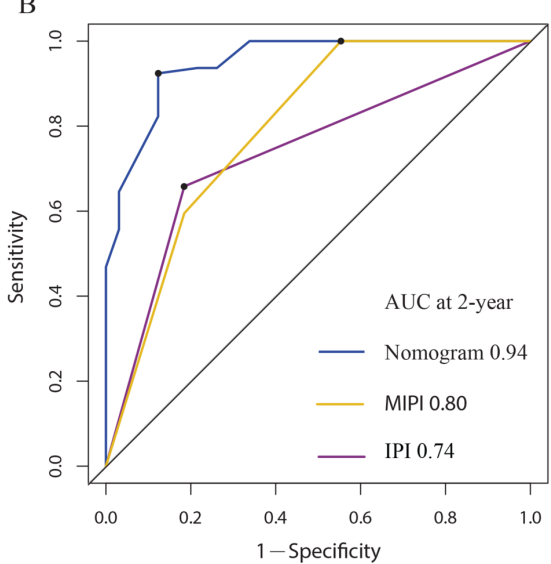

$\mathrm{C}$

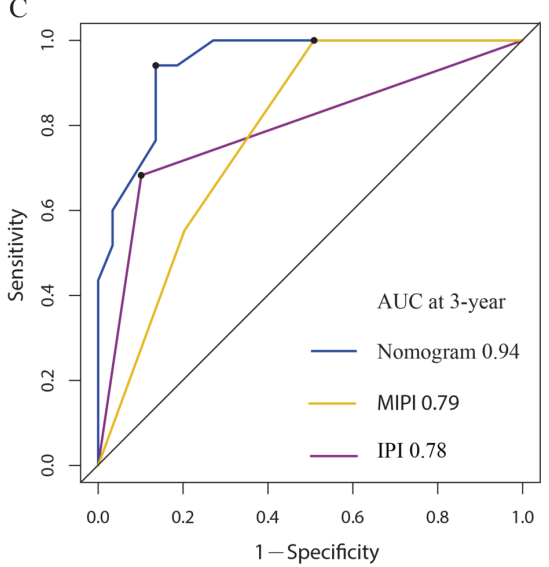

Figure 6 Time-dependent receiver operating characteristic curves from the nomogram, IPI and MIPI for 1 year (A), 2 year (B) and 3 year (C) overall survival in the validation cohort. AUC, area under the curve; IPI, International Prognostic Index; MIPI, Mantle cell lymphoma International Prognostic Index. 
This nomogram has several limitations. The main limitation of this study is the insufficient number of samples. Although the sample size in this study is fairly large for a local region, it remains insufficient for larger regions, not to mention globally. In addition, the nomogram was designed based on the local patient population. It remains uncertain whether the nomogram is precise for other populations or regions. The National Cancer Database of USA contains a large amount of cancer data that may provide conditions for further validation.

In conclusion, this nomogram might be helpful to allow individualized, risk-adapted treatment decisions in patients with MCL. The nomogram exhibited an improved ability for risk stratification compared with IPI and MIPI and would facilitate therapeutic decision-making and individualized patient counseling.

Correction notice This article has been corrected since it was published Online First. Xiao Zheng has been included as co-corresponding author.

Contributors $\mathrm{YZ}$ wrote the main manuscript text, $\mathrm{WX}$ provided detailed information of patients, XZ and ZZ constructed the nomogram and performed statistical analysis. All authors collaborated in the collection and interpretation of the data and contributed to the manuscript.

Funding This work was supported by grants from Jiangsu Key Laboratory of Medical Science and Laboratory Medicine (JSKLM-2014-003) and Changzhou Science and Technology Project (Applied Based Research, Nos. CJ20160021 and CJ20179025).

Competing interests None declared.

Patient consent Not required.

Ethics approval Ethics Committee of Third Affiliated Hospital of Soochow University.

Provenance and peer review Not commissioned; externally peer reviewed.

Data sharing statement The data sets used and analysed during the current study are available from the corresponding author on reasonable request.

Open access This is an open access article distributed in accordance with the Creative Commons Attribution Non Commercial (CC BY-NC 4.0) license, which permits others to distribute, remix, adapt, build upon this work noncommercially, and license their derivative works on different terms, provided the original work is properly cited, an indication of whether changes were made, and the use is non-commercial. See: http://creativecommons.org/ licenses/by-nc/4.0/.

\section{REFERENCES}

1 Dreyling M, Kluin-Nelemans HC, Beà S, et al. Update on the molecular pathogenesis and clinical treatment of mantle cell lymphoma: report of the 11th annual conference of the European Mantle Cell Lymphoma Network. Leuk Lymphoma 2013;54:699-707.

2 Smedby KE, Hjalgrim H. Epidemiology and etiology of mantle cell lymphoma and other non-Hodgkin lymphoma subtypes. Semin Cancer Biol 2011;21:293-8.

3 Goy A, Kahl B. Mantle cell lymphoma: the promise of new treatment options. Crit Rev Oncol Hematol 2011;80:69-86.

4 Tiemann M, Schrader C, Klapper W, et al. Histopathology, cell proliferation indices and clinical outcome in 304 patients with mantle cell lymphoma (MCL): a clinicopathological study from the European MCL Network. Br J Haematol 2005;131:29-38.
5 Hoster E, Dreyling M, Klapper W, et al. A new prognostic index (MIPI) for patients with advanced-stage mantle cell lymphoma. Blood 2008;111:558-65.

6 Han DS, Suh YS, Kong SH, et al. Nomogram predicting long-term survival after d2 gastrectomy for gastric cancer. J Clin Oncol 2012;30:3834-40.

7 Wang Y, Li J, Xia Y, et al. Prognostic nomogram for intrahepatic cholangiocarcinoma after partial hepatectomy. J Clin Oncol 2013;31:1188-95.

8 Yang L, Shen W, Sakamoto N. Population-based study evaluating and predicting the probability of death resulting from thyroid cancer and other causes among patients with thyroid cancer. J Clin Oncol 2013;31:468-74.

9 Bochner BH, Kattan MW, Vora KC; International Bladder Cancer Nomogram Consortium. Postoperative nomogram predicting risk of recurrence after radical cystectomy for bladder cancer. J Clin Oncol 2006;24:3967-72.

10 Touijer K, Scardino PT, staging Nfor. prognosis, and predicting treatment outcomes. Cancer 2009;115(13 Suppl):3107-11.

11 Shao Y, Ning Z, Chen J, et al. Prognostic nomogram integrated systemic inflammation score for patients with esophageal squamous cell carcinoma undergoing radical esophagectomy. Sci Rep 2015;5:18811.

12 Sabattini E, Bacci F, Sagramoso C, et al. WHO classification of tumours of haematopoietic and lymphoid tissues in 2008: an overview. Pathologica 2010;102:83-7.

13 lasonos A, Schrag D, Raj GV, et al. How to build and interpret a nomogram for cancer prognosis. J Clin Oncol 2008;26:1364-70.

14 Jares $\mathrm{P}$, Colomer $\mathrm{D}$, Campo E. Molecular pathogenesis of mantle cell lymphoma. J Clin Invest 2012;122:3416-23.

15 Navarro A, Clot G, Royo C, et al. Molecular subsets of mantle cell lymphoma defined by the IGHV mutational status and SOX11 expression have distinct biologic and clinical features. Cancer Res 2012;72:5307-16.

16 Bao WY, Wang Y, Hu XM, Xm H, et al. [Clinical features and prognostic analysis of mantle cell lymphoma]. Zhonghua Xue Ye Xue Za Zhi 2012;33:814-8.

17 Kang BW, Sohn SK, Moon JH, et al. Clinical features and treatment outcomes in patients with mantle cell lymphoma in Korea: Study by the Consortium for Improving Survival of Lymphoma. Blood Res 2014;49:15-21.

18 Romaguera JE, Medeiros LJ, Hagemeister FB, et al. Frequency of gastrointestinal involvement and its clinical significance in mantle cell lymphoma. Cancer 2003;97:586-91.

19 Vose JM. Mantle cell lymphoma: 2015 update on diagnosis, risk-stratification, and clinical management. Am J Hematol 2015;90:739-45.

20 Chihara D, Asano N, Ohmachi K, et al. Prognostic model for mantle cell lymphoma in the rituximab era: a nationwide study in Japan. Br J Haematol 2015;170:657-68.

21 Goswami RS, Atenafu EG, Xuan Y, et al. MicroRNA signature obtained from the comparison of aggressive with indolent non-Hodgkin lymphomas: potential prognostic value in mantle-cell lymphoma. J Clin Oncol 2013;31:2903-11.

22 Zeng Q, Xue N, Dai D, et al. A nomogram based on inflammatory factors C-reactive protein and fibrinogen to predict the prognostic value in patients with resected non-small cell lung cancer. J Cancer 2017:8:744-53.

$23 \mathrm{Kim} \mathrm{BH}$, Kim K, Chie EK, et al. Risk stratification and prognostic nomogram for post-recurrence overall survival in patients with recurrent extrahepatic cholangiocarcinoma. HPB 2017;19:421-8.

24 Xie H, Chen LM, Sun HH, et al. Long-term prognostic value of QRS score in patients with successfully recanalized coronary chronic total occlusion and construction of a predictive nomogram model. Int I Cardiol 2017;227:698-703.

25 Shi KQ, Cai YJ, Lin Z, et al. Development and validation of a prognostic nomogram for acute-on-chronic hepatitis B liver failure. J Gastroenterol Hepatol 2017;32:497-505.

26 Zheng Z, Li X, Zhu Y, et al. Prognostic nomogram for previously untreated adult patients with acute myeloid leukemia. Oncotarget 2016;7:71526-35.

27 Vernerey D, Huguet F, Vienot A, et al. Prognostic nomogram and score to predict overall survival in locally advanced untreated pancreatic cancer (PROLAP). Br J Cancer 2016;115:281-9.

28 Hoster E, Rosenwald A, Berger F, et al. Prognostic value of Ki-67 index, cytology, and growth pattern in mantle-cell lymphoma: results from randomized trials of the European Mantle Cell Lymphoma Network. J Clin Oncol 2016;34:1386-94. 